Anales de Geografía de la Universidad Complutense ISSN: 0211-9803

http://dx.doi.org/10.5209/AGUC.66942

\title{
La trama del lugar en los Erasmus: intersecciones geográficas a partir del caso de estudiantes latinoamericanos
}

\author{
José Ignacio Larreche ${ }^{1}$; María Belén Nieto ${ }^{2}$ \\ Recibido: 27 de octubre del 2017 / Enviado a evaluar: 28 de noviembre del 2018 / Aceptado: 17 de octubre del 2019
}

Resumen. El presente trabajo analiza las motivaciones, vivencias y percepciones de un grupo de estudiantes latinoamericanos en la ciudad de Lodz (Polonia) en el marco del programa Erasmus. A partir de una metodología cualitativa, y en base a entrevistas de carácter no directivo y la observación participante se indaga en las particularidades que supone ser un extranjero en la Europa de hoy y cómo la cuestión cultural imprime un juego dicotómico entre un nosotros y un ellos que se refleja en las trayectorias diarias de los latinos, desde la perspectiva geográfica de lugar. A lo largo de la investigación se repiensa el concepto de lugar y se logra concluir que la globalización educativa como fenómeno propio de la coyuntura actual conduce a plantear interesantes análisis del lugar partiendo de los estudiantes.

Palabras clave: Erasmus; estudiantes latinoamericanos; lugar; nosotros-ellos.

\section{[en] The place tissue: geographics intersections, the case of Erasmus latin students}

\begin{abstract}
This paper analyzes the motivations, experiences and perceptions of a group of Latin American students according to the Erasmus exchange program at Lodz, Poland. Based on a qualitative methodology, open interviews and participant observation, we research the effects of being a foreigner in today's Europe and how the cultural issue prints a dichotomy game between us and they, which is reflected in the daily trajectories of these students, from the geographical perspective of place. Throughout the article, the concept of place is deeply recognized and it is possible to conclude that the educational globalization is a social phenomenon which proposes interesting geopolitical and human geography results, around the subjects of analysis.
\end{abstract}

Keywords: Erasmus; latin students; place; us-they.

1 Departamento de Geografía y Turismo, Universidad Nacional del Sur (UNS) y Consejo Nacional de Investigaciones Científicas y Técnicas (CONICET). Bahía Blanca, Argentina.

E-mail: joseilarreche@gmail.com

2 Departamento de Geografía y Turismo, Universidad Nacional del Sur (UNS Bahía Blanca, Argentina).Centro de estudios sobre la Acción y el Desarrollo Territorial (ADETER).

E-mail: mbnieto@uns.edu.ar 
[fr] Le tissu de la place dans l'erasmus: intersections géographiques du cas des étudiants latino-américains

Résumé. Cet article analyse les motivations, les expériences et les perceptions d'un groupe d'étudiants latino-américains selon le programme d'échange Erasmus à Lodz en Pologne. Basé sur une méthodologie qualitative, des interviews ouvertes et des observations participantes, nous étudions les effets de l'étranger dans l'Europe d'aujourd'hui et comment le problème culturel imprime un jeu de dichotomie entre nous et ils, qui se reflète dans les trajectoires quotidiennes de ces étudiants alors une perspective géographique du lieu. Tout au long de l'article, le concept de lieu est profondément reconnu et il est possible de conclure que la mondialisation de l'éducation est un phénomène social qui propose des résultats géopolitiques et humains intéressants sur le plan de l'analyse.

Mots clés: Erasmus; les étudiants latino-américains; lieu; nous-ils.

Cómo citar. Larreche, J.I.; Nieto, M.B. (2019): La trama del lugar en los Erasmus: intersecciones geográficas a partir del caso de estudiantes latinoamericanos. Anales de Geografía de la Universidad Complutense, 39(2), 337-357.

Sumario. 1. Introducción. 2. Estado del arte y abordaje metodológico. 3. Los Erasmus latinoamericanos, un perfil sociodemográfico. 4. Las escalas del lugar. 4.1. La xenofobia como obstáculo del lugar. 4.2. Entre la Universidad y el Dorm: la inestabilidad del lugar. 5. Repensando el lugar. 6. A modo de conclusión. 7. Bibliografía.

\section{Introducción}

Los principales objetivos del programa Erasmus ${ }^{3}$, creado en 1987, han sido optimizar la calidad educativa superior y fomentar la movilidad internacional entre estudiantes del continente europeo. Sin embargo, la proliferación de convenios académicos con universidades de otras regiones ha abierto la posibilidad para que otros estudiantes no europeos realicen una estancia en dicho continente y también gocen de estos beneficios. Estas experiencias académicas suponen un encuentro con otras culturas o, en términos antropológicos, con el otro, que constituye una premisa a considerar en la comprensión de estas espacialidades del conocimiento (King y Raghuram, 2013).

De acuerdo a UNESCO el número de estudiantes que emigran de su país de origen con fines académicos se encuentra en un aumento progresivo, configurando una práctica cada vez más extendida entre la comunidad de universitarios. Por otro lado, es notoria la diversidad en la oferta de becas que se desprenden de la Unión Europea a partir del consorcio Erasmus: credit mobility, erasmus eurica, erasmus place, erasmus mundus son algunas opciones a nivel de grado, maestría, doctorado, doctorado sándwich, posdoctorado y/o de staff. Dicha iniciativa conlleva el involucramiento de más de cien universidades del mundo que establecen una red de nodos que engrosa el

3 EuRopean Community Action Scheme for the Mobility of University Students. 
fenómeno de la globalización educativa actual y, consigo, la importancia de la internacionalización en la estructura de las casas de altos estudios.

A partir de un estudio exploratorio este trabajo analiza las motivaciones, vivencias y percepciones de un grupo de estudiantes latinoamericanos en la ciudad de Lodz, Polonia. A pesar de que existen investigaciones vinculadas a la experiencia Erasmus, éstas se han focalizado en los estudiantes europeos siendo el registro de contribuciones referidas a estudiantes de otra procedencia una cuestión a compensar. En base a fuentes primarias y secundarias y con un fuerte énfasis del criterio cualitativo se persigue descubrir los efectos de una identidad extranjera en la Europa de hoy que se reflejan en las trayectorias diarias de los latinoamericanos desde una perspectiva geográfica humanista. Esta vertiente nos resulta especialmente interesante dada las confusiones a las que invita el concepto de lugar. Es por ello que abordaremos al lugar como una noción abierta a múltiples derivas precisadas por el caso empírico.

La estructura del texto contempla cuatro partes. Inicialmente se presentan los lineamientos de partido en torno a la temática en conjunto con el encuadre metodológico. Seguidamente se expone las principales características de los sujetos de análisis, a modo de perfil. El trabajo intenta crecer en complejidad en la tercera parte desde donde se desprenden las distintas escalas del lugar para discutirlas teóricamente en el apartado llamado "repensando el lugar". Por último se despliega un cierre a medias ya que invita a proseguir rastreando más de estas cuestiones.

\section{Estado del arte y abordaje metodológico}

A pesar de que existen investigaciones vinculadas a la experiencia erasmus, éstas se han focalizado en explicar las características de las movilidades de estudiantes europeos (González et al., 2011; Bótas y Huisman, 2013; Cairns, 2014), dejando una laguna de conocimiento en el registro de contribuciones similares referidas a estudiantes no europeos. En este apartado, se hará hincapié en algunos aportes teóricos que serán útiles al momento de enmarcar las discusiones venideras.

El aporte de King y Raghuram (2013) considera la relación entre conocimiento y espacialidad. Estos autores incorporaron el análisis de los estudiantes como un componente del proceso de migración ${ }^{4}$ y, desde una visión geopolítica, repararon en los múltiples roles que éstos adoptan en el contexto actual como potenciales trabajadores, miembros de familia (a través del envío de remesas) o solicitantes de

\footnotetext{
${ }^{4} \mathrm{Si}$ bien en función de respetar los desarrollos de los autores se emplearon las palabras exactas de la fuente, se presta debe aclarar que no podemos hablar de un proceso de migración pero sí de movilidad como concepto genérico ya que la migración requiere un establecimiento definitivo en el espacio de destino y esto está terminantemente denegado a partir de la temporalidad de los visados de estudiantes latinoamericanos.
} 
asilo. Un tema cardinal que concluyeron en su investigación estriba en la contradicción que emerge entre estudiantes internacionales "deseados" y los "no deseados", en base a la política de control post 11 de septiembre que los ha reducido y jerarquizado estereotipadamente al ingresar a los países del hemisferio norte. En su explicación proponen el concepto de "espacialidades del conocimiento" que postula que estos estudiantes no generan simplemente conocimiento in situ, a pesar de que muchas veces son activamente reclutados ${ }^{5}$ para el beneficio de la ciencia del país que los alberga, sino también en la producción de conocimiento cuando regresan a su institución de origen. De esta forma, la adquisición cognitiva y las técnicas aprehendidas quedan en el individuo traspasando fronteras estatales. Así, "el conocimiento es reconocido como una extensión internacional, ya que necesita ir más allá de los estrechos confines" (King y Raghuram, 2013:133).

En segundo lugar, Teichler (2004) postuló lineamientos generales de los intercambios Erasmus en el exterior, y esto fue considerado al realizar un contrapunto con el caso de los estudiantes latinoamericanos. Este autor esgrime que la movilidad transnacional constituye una "opción normal" para los estudiantes europeos y da cuenta de las principales razones para emprenderla. Por otro lado, aportó detalles sobre el proceso de aplicación, evaluación y selección que vincula a estos programas con una dimensión que denomina "movilidad vertical", referida a la pretensión de los estudiantes en conseguir cursos mejores cualificados que el de sus universidades de origen, aducido a partir de la garantía de éxito en el futuro de sus profesiones, sostenido en el prestigio que brinda Erasmus. Completa esta idea con la de "movilidad horizontal", entendida como los intercambios culturales significativos que se producen entre estudiantes extranjeros, es decir, la fase social de la espacialidad del conocimiento que trastocará la totalidad de la experiencia en un sentido positivo o negativo dado que se trata, en general, de una incursión en tierras incógnitas.

Otro artículo pertinente fue brindado por Cairns (2014) centrado en el caso específico de los estudiantes irlandeses. La revisión del mismo recae fundamentalmente en el hecho de que la República de Irlanda se posiciona como una economía secundaria a causa de una reciente recesión. Esto impacta en la decisión de los estudiantes locales de partir a centros de estudio que vaticinen un mejor devenir en el mercado de trabajo de su país ${ }^{6}$. La condición económica de la mayoría de los países de Latinoamérica está signada por fuertes ciclos de inestabilidad que justifican y aceleran la necesidad de obtener una ventaja competitiva fuera de la región que mejore las credenciales académicas. Cairns titula su obra "I wouldn't stay here" ("no me quedaría aquí") para reflejar la incertidumbre que suscita el presente y el futuro de

\footnotetext{
${ }^{5}$ Según la RAE la acción de reclutar significa reunir gente con un propósito determinado. El recluta es un actor del ámbito militar, que debe sortear distintas etapas antes de ser reclutado, a modo de un paralelismo crítico, lo mismo pasa con los estudiantes que logran ser seleccionados para sus becas.

${ }^{6}$ Se debe recordar que la nación irlandesa, ante la crisis que afecta a la Unión Europea en 2008, fue catalogada como una de los PIGS (en español cerdos) junto con Portugal, Grecia y España.
} 
estos jóvenes irlandeses, un antecedente que puede brindar herramientas para pensar un aspecto de la realidad en nuestro recorte.

Por último otras contribuciones que dialogan con el cometido del presente trabajo están nucleadas en la prestigiosa revista Journal of Geography in Higher Education y atienden la cuestión del otro en la experiencia educativa (Monk, 2000), las implicancias de ciudadanía política (Llurda et al., 2016) y estudios de caso como el irlandés (Deakin, 2014; Iriondo, 2019).

La presente investigación posee un enfoque cualitativo para comprender las singularidades de ser un extranjero en la Europa de hoy a partir de las trayectorias de un grupo de estudiantes latinoamericanos durante su estancia en Polonia. Para Marshall y Rossman (1999, en Vasilachis de Gialdino, 2006) la investigación cualitativa es pragmática, interpretativa y está asentada en la experiencia de las personas. Se trata de una amplia aproximación a los fenómenos sociales donde el trabajo de campo se presenta como indispensable para su consecución y más precisamente la entrevista en profundidad (Sautu et al. 2005; Mendicoa, 2003), que permite analizar detalladamente las vivencias de los estudiantes seleccionados. Considerando que a partir de la competencia narrativa se expresan las necesidades, los deseos, las preocupaciones y los sentimientos, el análisis de los discursos permite reflexionar acerca de las percepciones individuales en base a interacciones que acaecen en tres tipos de espacios solapados entre sí: el espacio social de Polonia; la universidad y las residencias estudiantiles donde estaban alojados.

Los sujetos de análisis fueron trece de distinta procedencia y con paridad en cuanto al género; los datos fueron sustraídos mediante breves cuestionarios para establecer un perfil, entrevistas de carácter no directivo (abiertas y semidirigidas) y la observación participante, complementados con registros de blogs y otras actividades del círculo Erasmus que tuvieron lugar durante el período de análisis (desde septiembre 2016 hasta marzo 2017). La observación participante involucra la interacción social entre el investigador en el mileu de los investigados, y durante la cual se recogen datos de modo sistemático y no intrusivo pudiéndose ajustar mejor a la realidad (Taylor y Bogdan, 1986 en Santarelli y Campos, 2002). Es por ello que la naturaleza de la presente investigación requiere especificar el contexto de la observación dado que uno de los autores ha adoptado igualmente el rol de estudiante latinoamericano. En este sentido, la observación se produjo de forma planificada pero principalmente espontánea ya que la convivencia con el resto de los estudiantes ha sido continua. La misma fue realizada en la asistencia a los cursos tomados en la universidad, los espacios compartidos en la residencia estudiantil así como también en fiestas nocturnas y diferentes viajes grupales a ciudades y países europeos realizados en el transcurso de los meses de beca. En este sentido Rosana Guber explica que tradicionalmente el objetivo de la observación participante "ha sido detectar los contextos y situaciones en los cuales se expresan y generan los universos culturales y sociales, en su compleja articulación y variabilidad" (2004, p. 115). En este caso los universos culturales serán puestos al servicio del concepto de lugar desde la ciencia geográfica. 
Este doble rol de investigador e investigado (por las afinidades con los sujetos de análisis en términos de estudiante extranjero) tuvo dos momentos conectados: un primer momento que inspiró una preocupación casi insospechada en el devenir de la estancia académica y un segundo momento más reflexivo en donde la preocupación se convirtió en un problema de investigación después de retornar. El distanciamiento permitió un desacople (relativo) de la implicación de los pensamientos y valoraciones que han sido confiados. Con esto queremos demostrar lo intrincado de la investigación social ante la continua filtración de la subjetividad mucho más cuando la pregunta parte de una experiencia personal.

\section{Los Erasmus latinoamericanos, un perfil sociodemográfico.}

La movilidad estudiantil acarrea un efecto de identidad disociada en términos geográficos, como la que emerge en cualquier desplazamiento fuera de las fronteras nacionales, ante la experiencia de sentirse extranjero, extraño. Este rasgo es un denominador común, inclusive para los estudiantes europeos que, sin recorrer grandes distancias como sus pares sudamericanos o asiáticos, también pueden sentirse extraños en otros países de su continente. Pero más allá de esa doble pertenencia, ser erasmus latinoamericano representa una condición por lo menos novedosa ${ }^{7}$. A fines de la investigación, se consultó a estudiantes de Argentina, Bolivia, Chile, Colombia, Ecuador, El Salvador, Guatemala, Honduras, Nicaragua, Paraguay, Perú y México.

El primer dato importante que se evidencia es el alto porcentaje de estos estudiantes que nunca han estado antes en Europa (89\%), lo que permite pensar que la oportunidad académica envuelve, muchas veces, un mecanismo único para viajar al exterior y ser los primeros en emprender dicho desplazamiento en el seno de sus respectivos núcleos familiares. Las coyunturas político-económicas, sumadas a las distancias geográficas y a la fuerte polarización social, características propias de los países de esta región, son dificultades estructurales para estos estudiantes que, sin la cobertura de la beca, no podrían costear una travesía de aproximadamente 2000 euros $^{8}$. Por lo tanto, residir por un semestre o un año en Europa, de acuerdo al caso, es una situación totalmente nueva y podríamos aseverar que dicha movilidad no forma parte de su "habitus", en contraste con lo que expresa Teichler (2014) cuando analiza

\footnotetext{
${ }^{7}$ Erasmus Mundus fue el primer programa en incorporar a instituciones de América Latina desde el año 2010 hasta el año 2017.

${ }^{8}$ Se tomó como referencia la tarifa final de la ruta realizada por un estudiante argentino, punto más austral del Cono Sur de América.

${ }^{9}$ En forma sintética, la teoría bourdiana define el "habitus" como el sistema de disposiciones duraderas y transferibles, estructuras estructuradas predispuestas para funcionar como estructuras estructurantes, es decir, como principios generadores y organizadores de prácticas y representaciones (Bourdieu, 1991). De este modo, si bien el habitus es variable con el tiempo y la capacidad de agenciamiento de los sujetos, el peso de las primeras experiencias reviste un carácter duradero, en los sujetos del estudio.
} 
a los estudiantes europeos. A esto se le adiciona el trayecto aéreo (desde América del Sur o América Central a Europa), que implica también un estreno a bordo de un avión (77\%) que puede conllevar a situaciones de estrés y cansancio, causados por la duración del viaje. Por ejemplo, el vuelo desde Buenos Aires a Varsovia con una escala de conexión en Alemania tiene una duración total de $14 \mathrm{hs}$, de lo que se desprende que también son principiantes frente a maniobras de embarques, conexiones, controles de seguridad, y barreras idiomáticas que sus pares europeos conciben como rutinarias.

Figura 1. Universidades socias del programa Erasmus Eurica

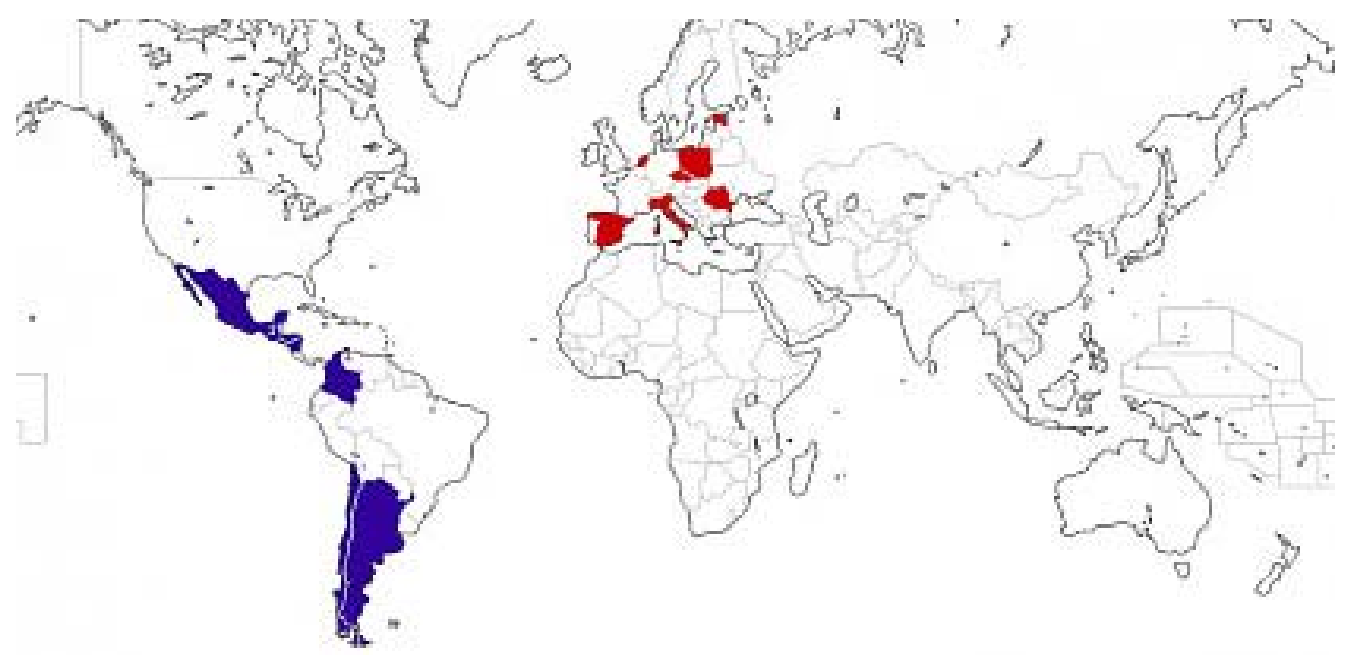

Fuente: www.eurica.nl, 2017.

Es importante señalar que casi la mitad de estos estudiantes permanecerán un año, y el resto un semestre bajo una "movilidad crediticia", lo que significa que el programa se completa cuando los estudiantes regresan a la institución de origen (King y Raghuram, 2013), entre las que se encuentran la Universidad Nacional del Sur, Universidad de San Carlos de Guatemala, Universidad de Magdalena, Universidad Nacional Autónoma de Honduras, Universidad Nacional Autónoma de Nicaragua, Universidad de El Salvador, Universidad del Centro, Universidad de La Serena, Universidad Autónoma del Estado de Hidalgo, entre otras (ver figura 1). La decisión de estar en el exterior se apoya en los siguientes factores: duración de la beca, utilidad de los cursos (acreditables en la universidad de origen), el monto de la beca y el país 
de destino ${ }^{10}$. En el artículo de Teichler (2004), los estudiantes europeos declaran que las metrópolis son determinantes en sus opciones finales, como Roma y París, o prefieren pequeñas ciudades universitarias de perfil más tradicional como Salamanca y Tübingen, evaluando además el clima como motivación de su estancia.

En el presente estudio, la ciudad de Lodz fue seleccionada fundamentalmente por convenios institucionales entre las casas de estudio que se asocian al programa $(62,5 \%)$, lo que sugiere que la libertad de elección estuvo condicionada por la oferta que no resultó tan amplia ${ }^{11}$. En el caso de los estudiantes europeos, los convenios contemplan una gran variedad de universidades de los países miembros de la Unión Europea. Por otra parte, el $20 \%$ de los entrevistados indicó que el prestigio de la facultad y la calidad de los cursos, la menor competencia entre los candidatos locales para la adjudicación de plazas y la historia de Polonia, fueron otros factores que influyeron en la decisión final.

Con respecto a los objetivos de la movilidad, estos son similares a los de sus homólogos europeos: practicar un idioma extranjero (inglés) y obtener una experiencia educativa de alta calidad fueron los que más se repitieron a pesar de que también se vislumbró la importancia e interés que reposa en el intercambio cultural: "aprender y compartir otras costumbres y estilos de vida". De esta manera, se corrobora que los estudiantes "esperan los beneficios académicos, culturales, lingüísticos y profesionales, pero no les importa combinarlos con una vida extracurricular interesante durante el período que pasan en el extranjero" (Teichler, 2004: 397).

Otro factor particular de ser Erasmus latinoamericano se apoya en el proceso de evaluación de los postulantes, dispar con el esquema de los candidatos del viejo continente. Mientras el cuello de botella es sustancialmente menor de lo que se podría suponer en el caso europeo (Teichler, 2004), los latinoamericanos deben atravesar diversas exigencias como parte de los requisitos formales de aplicación, entre los que aparecen el promedio de calificaciones, las recomendaciones certificadas de los profesores en dos idiomas, la acreditación de niveles de lengua extranjera y la elaboración personal de cartas de motivación. Si a eso le sumamos los avatares burocráticos post-selección, primordialmente los visados, podemos determinar que se trata de una aplicación compleja que requiere tiempo y planificación. Asimismo, a diferencia de lo que sucede con los europeos, el número de latinoamericanos

\footnotetext{
${ }^{10}$ King \& Raghuram (2013) indican que existen asimetrías de género que afectan la decisión de algunas estudiantes de ir al extranjero debido a las normas patriarcales en sus sociedades de origen. Los lazos familiares y el rol doméstico de la mujer en los países latinoamericanos podrían seguir siendo centrales y pueden influir en estas posibilidades de movilidad.

${ }^{11}$ Para citar un caso, el programa Erasmus Eurica posee sólo 6 universidades europeas (España, Polonia, Holanda, República Checa, Estonia e Italia) como socias lo que reduce en gran medida las opciones académicas de los candidatos.
} 
beneficiados por las becas son pocos y éstos accederán a estipendios "muy buenos" según expresaron, una vez en el destino ${ }^{12}$.

\section{Las escales del lugar}

King y Raghuram afirman que "las experiencias de movilidad variarán según el nivel de estudio, el tema de estudio, el tipo de actividad y el país de destino" (2013:130). En este sentido, entendemos que esta experiencia de movilidad tiene otra implicancia vertebral para la geografía humana. Erasmus es una experiencia singular para la mayoría de los estudiantes ${ }^{13}$ y esto nos lleva a repensar: ¿cuáles son los alcances psicosociales de la misma?, ¿es una efectiva oportunidad cultural (movilidad horizontal) de encuentro con estudiantes de diferentes partes del mundo?, ¿es una estancia que impone sólo dificultades académicas compensadas por un prestigio mayor (movilidad vertical)? Más aún, ¿qué valoraciones aparecen en torno a Polonia? ¿Qué relaciones existen entre el contexto actual del continente europeo (tras la crisis de los refugiados y el BREXIT) y las vivencias de estos estudiantes extranjeros en la conformación del lugar?

Empezaremos a intentar esbozar algunas respuestas, a partir de comprender los principales retos, sentimientos y emociones que le confieren estos sujetos a la experiencia erasmus, estructurada en tres espacios de socialización que parten de la ciudad como escala general (características de Polonia, actividades cotidianas como compras, trámites, vida nocturna, viajes), siguiendo por los aspectos sociales en el seno del campus universitario (relación con compañeros, oficina de asuntos internacionales) y culminando en la convivencia dentro del edificio residencial (los impactos de la convivencia, vínculos con los recepcionistas). Es decir, se discutirá acerca del sentido de lugar de los estudiantes latinoamericanos que engloba itinerarios más públicos y otros de índole más privada; señalando hechos que contribuyan a fortalecer o precarizar el lugar, entendido como "el espacio más el valor humano" (Maltas, 2015:205).

La interiorización en torno al concepto de lugar se ubica a fines de la década de 1970 en la corriente que se conoce como geografia humanista. Barros (2000) concibe al lugar como una porción concreta del espacio con una especial carga simbólica y

\footnotetext{
${ }^{12}$ Las cifras de estipendios son públicas en las páginas correspondientes a los distintos programas que financia la Universidad de Groningen, como sede central. En numerosas ocasiones, los estudiantes latinoamericanos expresaron que durante sus charlas con sus pares europeos, éstos manifestaban quejas al detectar que a los primeros se les pagaba más que a ellos. Para un mejor entendimiento confrontar con Otero (2008) The socio-economic background of Erasmus students: A trend towards wider inclusion.

${ }^{13}$ En la red social Facebook, existe un famoso grupo llamado Erasmus Bible ("La Biblia Erasmus") en donde se plasman distintas frases, fotos, actividades y videos que permiten ver la magnitud que representa para el sector estudiantil acceder a este intercambio.
} 
afectiva. Por su parte Maltas (2015) asume que el lugar halla su definición en relación con la noción de límite (bound), limitación (limit) o superficie. En una misma línea lo que plantea Barros es que la idea de lugar está asociada no sólo a valores y significados sino a comunidades específicas, eso lo distancia y diferencia del concepto genérico de espacio.

En otro orden de ideas Tuan (1977) revela que el lugar hace referencia a espacios delimitados, con límites precisos que para los sujetos representan certezas y seguridades otorgadas por lo conocido. Desde estos puntos de vista, los latinoamericanos se ubicarían como los outsiders o "periféricos" (Barros, 2000) de acuerdo, por un lado, a los límites que impone la visión del lugar de la comunidad anfitriona y, por otro lado, al grado de conocimiento que poseen del sistema cultural y su funcionamiento: el lugar se funde en tanto se estrechan las costumbres, la historia y la memoria de una comunidad relativamente homogénea: "creo que si vuelvo, nunca me quedaría en Polonia...existen muchas diferencias que no hacen fácil la adaptación", relata una estudiante latinoamericana.

\subsection{La xenofobia como obstáculo del lugar}

Lodz es una ciudad del centro de Polonia que posee aproximadamente 700.000 habitantes y constituye la tercera ciudad más populosa a nivel nacional.

Figura 2. Área de estudio.

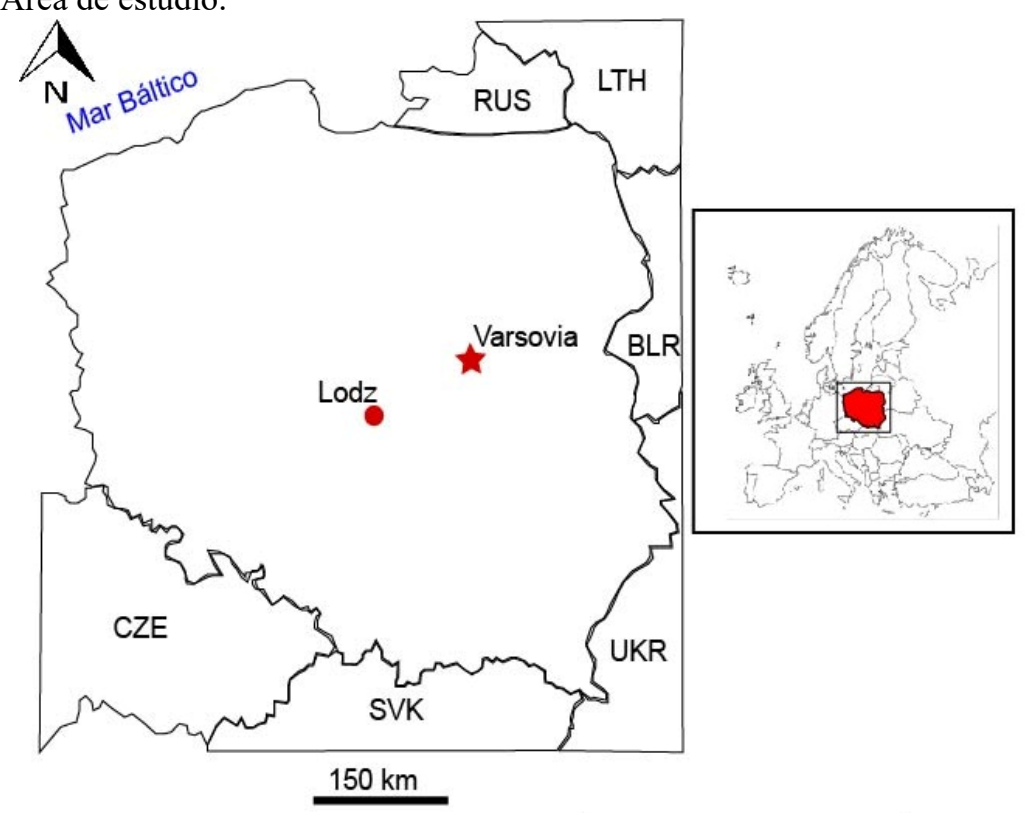

Fuente: Larreche, 2017. 
El contraste cultural entre individuos de América Latina y los ciudadanos de la Europa Oriental es esperable, pero nuestra tarea se abocó a precisar el peso de esas diferencias al diseñar un lugar en términos fenomenológicos, para el caso de los recién llegados. Más de la mitad de los entrevistados reconocieron la lengua polaca como la barrera más importante en su adaptación; otros indicaron que el clima, la comida y "la sociedad polaca" fueron otros derroteros en sus trayectorias cotidianas. El respeto por las luces del semáforo, los horarios para comer y el número de comidas, la extensión de salidas nocturnas ("se sale y se regresa mucho más temprano de las fiestas") y la falta de ingredientes para cocinar ("no encuentro mis frijoles") son algunos hábitos en los que no coinciden.

Por otro lado, los desplazamientos por la ciudad están fuertemente controlados, "los oficiales de la MPK están en todos lados" aseguran varios entrevistados. Se trata de los tradicionales "controladores" de tickets, quienes se dispersan selectivamente por la ciudad ya que "están concentrados en las zonas de mayor consumo como el centro comercial Manufaktura pero también en la zona universitaria", concluye un estudiante multado. La vigilancia se agudiza en los "trams" y buses, donde continuamente se aplican infracciones a los estudiantes extranjeros pero también a la población local. No obstante, los entrevistados enfatizan el comportamiento xenófobo de éstos, que se muestran con una intención deliberada por castigar a los extranjeros: "me indigna porque el policía entra y lo primero que hace es dirigirse hacia mí porque tengo tez oscura o pelo negro".

Otro punto que atenta contra el espacio vivido es el crudo invierno, la nieve resulta desconocida para muchos estudiantes de los países latinoamericanos (especialmente de América Central) lo que representó un reto a su ánimo y bienestar y también para sus finanzas, por la compra de indumentaria específica que no seguirán usando en sus países de origen; el factor gastronómico puede resultar desapercibido en la construcción del lugar pero tiene un peso muy visible en el caso de los estudiantes en cuestión: "no hay variedad de frutas", "extraño la comida de mi país", "el café sabe mal aquí".

En relación a la sociedad polaca, esta es percibida como "fría", "pegada a las reglas", "silenciosa", "conservadora" y "demasiado católica" desde los parámetros de los latinoamericanos. Varios estudiantes fueron agredidos durante su estancia en Lodz. Queremos rescatar el episodio de uno de dos becarios que fueron intimidados en el espacio público expresando desconocer el motivo de la agresión aunque dejan entrever un modus operandi estimulado por la xenofobia: "yo pienso que ellos son agresivos con cualquiera que sea distinto". Un acontecimiento particular que da cuenta de esta xenofobia, en el período de análisis, tuvo lugar en el marco de la celebración del Día de la Independencia de Polonia (11 de noviembre), cuando se recomendó, por petición de las autoridades de las oficinas estudiantiles internacionales (y algunos profesores) que ningún estudiante extranjero participe del evento, debido a la presencia de grupos ultra-nacionalistas (lo que los medios difundieron como "La Marcha Patriótica") que podían poner en peligro su integridad física. Esto no constituye un hecho aislado, dado que en ese contexto tuvieron lugar 
importantes movilizaciones como respuesta a esta tendencia conservadora del gobierno: por ejemplo, el "lunes negro" protagonizado por ciudadanas polacas a favor del aborto, protestas del Comité de la Defensa Democrática (KOD son sus siglas en polaco) contra las medidas antidemocráticas del Ejecutivo ${ }^{14}$, como las más notables.

La visión del consorcio Erasmus es la de "impulsar la movilidad intraeuropea de los estudiantes que, a su vez, es parte de un proyecto más amplio: el de crear un sentido compartido de pertenencia a través de los países europeos, especialmente, de la UE" (King y Raghuram, 2013:130). Sin embargo, esta prerrogativa puede ser pensada desde un patrón implícitamente eurocéntrico, ya que fue elaborada cuando los participantes de estas movilidades eran sólo los de dicho origen. Ante la incorporación de universidades de otras latitudes ¿dónde se alude a esos otros estudiantes?, ¿cómo se los integra? Suscribimos que en el contexto geopolítico actual, éstos se están convirtiendo en blanco de una creciente sospecha y, en esta perspectiva, Collins (2009) señala que los "no europeos" están negociando simultáneamente la inclusión y la exclusión en partes iguales. El "discurso de la racialización” aparece con frecuencia en los medios de comunicación y difusión repercutiendo en efectos psicosociales negativos para los estudiantes latinoamericanos en aumento de su sensación de topofobia (miedo al lugar).

Es preciso amplificar las experiencias de los becarios en torno a las infraestructuras de transporte que emplearon en sus viajes a distintas ciudades, aprovechando la posición geográfica de Lodz. La presencia de policías y fuerzas de seguridad militar vuelve a reproducirse tanto en trenes como buses según lo explicado, a pesar de que el control más exhaustivo se produce en los aeropuertos. Justamente, en esta última vía, se denota un rasgo más de esta globalización selectiva, donde aparece la jerarquía de los visados análoga a la jerarquía de las nacionalidades. La mayoría de los estudiantes latinoamericanos emplearon aeropuertos secundarios por un motivo de conveniencia económica, no obstante, las pautas de control, en muchos casos, terminaron siendo peores. Esto se corresponde con un menor flujo de turistas y por ende, menor posibilidad de que los extranjeros sean vistos envueltos en episodios de tratos evidentemente injustos por parte del personal aeroportuario. Todos los entrevistados declararon haber tenido al menos un problema en alguno de los aeropuertos que atravesaron durante sus viajes, desde una demora injustificada hasta un interrogatorio en la oficina de la policía del aeropuerto. Previo a dichos viajes, todos los becarios se asesoraron acerca de los requisitos formales de entrada al país de acogida, pero al momento del embarque, los controles de seguridad se vivenciaron

\footnotetext{
${ }^{14}$ Muchos profesores expresaron su preocupación durante los cursos en la Universidad de Lodz ante este panorama político y social que puede repasarse en muchos artículos periodísticos: http://www.eldiario.es/desalambre/Polonia-ultraderecha-xenofobia-rechazarefugiados_0_452505040.html en donde se puntualizan otros ataques a extranjeros. Un estudiante sirio en Poznan y un músico chileno en Varsovia fueron víctimas de fuertes golpizas en este período, por citar algunos. Esto nos remarca la fuerte influencia de la coyuntura geopolítica ante la configuración del lugar para estos estudiantes que se encarnan como objetivos de discriminación.
} 
incómodamente ante la revisión de sus pertenencias de forma reiterada. Esto quiere decir que los aeropuertos son nodos esclarecedores de la relación de poder asimétrica que sella la lógica global: aquellos que pierden son los ciudadanos al margen de la Unión Europea, debiendo formar en otra fila, soportar la mirada evaluadora, repetir constantemente sus razones de viaje, justificar la naturaleza de sus pertenencias y exhibir cada uno de los documentos probatorios de identidad, garantizando permanencia transitoria y fondos suficientes. Estos rituales son expuestos ante la comunidad de pasajeros que ganan, los que portan el color del pasaporte rojo. Asimismo, la portación de cara (demasiada barba, tez oscura, vestimenta oriental) es un dispositivo vigente $\mathrm{y}$, penosamente frecuente en el control de las salidas, reafirmando una actitud xenofóbica que vulnera en sentido doble a estos estudiantes, ya que se trata de jóvenes que pisan por primera vez Europa y lo hacen solos o en pequeños grupos portando el estigma de extranjeros. Como sostiene Bhabha "el aparato de seguridad da cuenta de los límites de la alteridad de la nación" (2013:51).

\subsection{Entre la universidad y el dorm $^{15}$ : la inestabilidad del lugar}

Esta sección dedicará su análisis al conjunto de factores alineados con la experiencia desde un ángulo académico pero también poniendo en tensión la dimensión sociocultural acaecida en la residencia estudiantil.

Las plazas a cubrir por estos estudiantes se repartieron de acuerdo a distintos niveles de formación, siendo la mayoría becas de grado y sólo dos de posgrado. Para favorecer el entendimiento de las vivencias alrededor del mundo universitario de estos becarios, se tuvo en cuenta cuestiones relativas a los mentores, los cursos, el vínculo con los compañeros polacos, entre otros. Los mentores, grupo de estudiantes avanzados polacos, tienen la función de auxiliar a los recién llegados en su inserción en la universidad y también en el acondicionamiento en la ciudad: inscripción a los cursos, apertura de cuenta bancaria, indicación de lugares importantes durante la estadía u otras demandas de los extralocales. Los entrevistados los percibieron por debajo de las expectativas porque, en algunos casos, como los estudiantes de Guatemala y Argentina, no había mentores designados por lo que tuvieron que recurrir a la ayuda de terceros o en otros casos su ayuda fue insignificante.

La oficina internacional de estudiantes (ISO) es otro punto interesante de la experiencia. En torno a ésta, surgen los puntos positivos del grupo. Algunas de las razones se fundamentaron en los eventos organizados en conjunto con los mentores para la comunidad de estudiantes extranjeros como barbacoas de bienvenida, los almuerzos de Navidad, los tótems para practicar una lengua extranjera, viajes organizados a ciudades polacas, juegos deportivos, premios Erasmus y fiestas nocturnas. Una mención especial merece el proyecto "University Diversity"

\footnotetext{
${ }^{15}$ Dorm es una palabra empleada por el común de los estudiantes para referirse a la residencia estudiantil.
} 
(Universidad Diversidad), la cual resaltaba la importancia de los valores culturales de los estudiantes visitantes (figura 3). Este concurso en especial posicionó a los extranjeros como protagonistas. Los estudiantes latinoamericanos que se animaron a posar fueron tratados como verdaderas estrellas de televisión: fueron maquillados, fotografiados con juego de luces y citados en lugares específicos. De todas las tomas, los estudiantes elegían una que se convertía en el banner tamaño real que iba a ser exhibido en el principal shopping de la ciudad (Manufaktura) junto a la bandera de su país y una breve descripción de sus biografías personales. Dicha iniciativa impactó positivamente y, como era opcional, generó que muchos de los que no habían participado insistieran por una segunda parte.

Figura 3. Iniciativa "University Diversity".

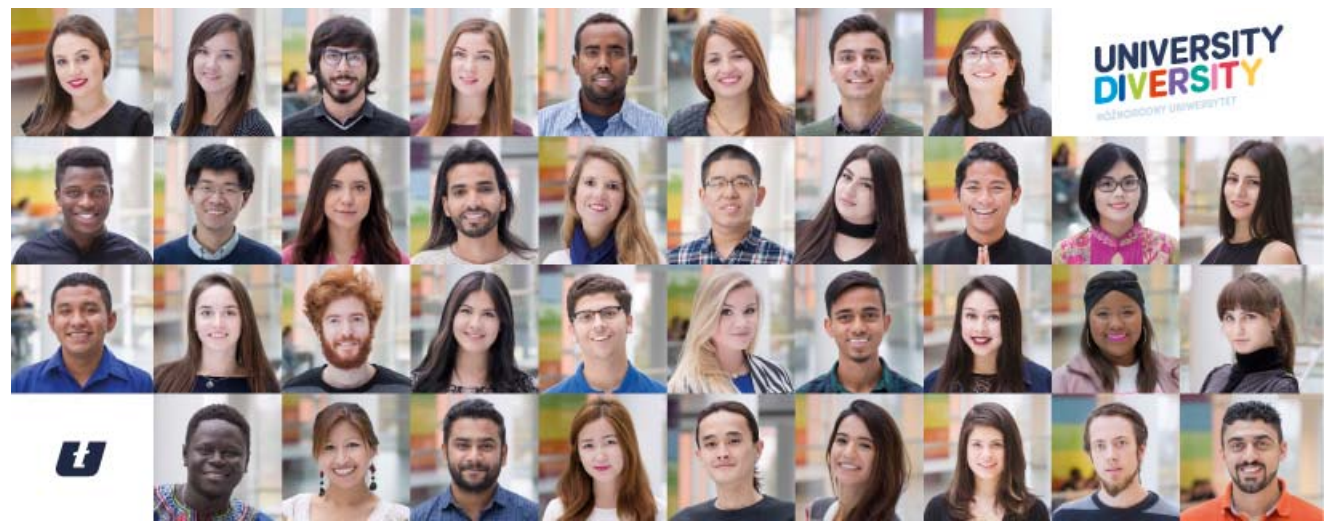

Fuente: Oficina de Relaciones Internacionales de Lodz, 2016.

Por otro lado, es necesario abocarnos al tema de los cursos. En el contrato formal de la beca los estudiantes firman en acuerdo con la aprobación de un número determinado de créditos (que reporta cada curso) para cumplir con los términos que exige la movilidad. En la mayoría de los casos los estudiantes latinoamericanos expresaron un desajuste entre las exigencias de la universidad de origen y la de Lodz, a pesar de que los cursos se impartan en inglés. En general, sus rendimientos en la Universidad de Lodz son "muy buenos", por lo que disponen de mayor tiempo libre en comparación con el ritmo académico de su país natal. Asimismo, estas performances satisfactorias los hace revalorizar la educación de su país y, si bien, los créditos de los cursos están pensados para que sean compatibles entre ambas instituciones, no se podría inferir características de una "movilidad vertical" (Teichler, 2004) dado que no reportan impactos de gran relevancia en comparación con lo esperado: "la verdad es que los contenidos son muy básicos" explica un becario. Por otro lado algunos de los entrevistados pudieron anotarse en cursos inexistentes en sus universidades de origen que ponderaron como "novedosos" e hicieron uso de bibliografía específica correspondiente a su especialidad en idioma original. 
Otro rasgo vinculado que merece atención es la vigilancia que se ejerce al momento de ingresar a las bibliotecas de cada facultad, incluida la central. En la entrada de cada una, las pertenencias de los estudiantes son confiscadas por personal de seguridad. A su vez, cada estudiante debe gestionar individualmente una clave de wi fi y dejar sus abrigos y pertenencias accesorias en un lock general de forma obligatoria. Esto ralentiza los tiempos y genera molestias entre los estudiantes además de que en muchas ocasiones el personal no docente hablaba en inglés.

El conflicto que más nos interesa para entender la noción de lugar en el ámbito universitario se presentó en la relación con el estudiantado polaco. Cinco de los entrevistados evaluaron la relación con sus compañeros de clase por debajo de las expectativas a los que asociaron como "cerrados" e "indiferentes". Cuando se consultó sobre las relaciones de compañerismo con otros estudiantes no polacos, más de la mitad reconocieron que sus contactos con estudiantes africanos o asiáticos habían sido escasos y la mayoría adujo intercambios más regulares con otros europeos occidentales, principalmente de España, Francia e Italia.

En segundo término, parar la profundizar en la complejidad del lugar también es importante ocuparse del espacio residencial. Esta esfera coincide con los límites de la vivienda que alude a sensaciones como la seguridad, comodidad, tranquilidad y, en algunas circunstancias, posee el mote de refugio ${ }^{16}$. Los entrevistados se alojaron en el campus Matejki, oficializado como "The Babel Tower"", uno de los complejos de dormitorios bajo la gestión de la Universidad de Lodz. Producto de la observación participante y las charlas informales con estudiantes de otros continentes, se determinó que allí sólo residen becarios de las regiones de América Latina, África, Asia, ucranianos y albaneses. En contrapartida, un gran número de españoles, turcos y rusos residían en los dormitorios de Lumumby que se encontraba a poca distancia del anterior y en donde "había menos comodidades".

Esta distribución geográfica materializa una segregación que contribuye a conformar guettos internos, cuestión que obstaculiza la idea de la movilidad horizontal: los latinoamericanos comparten habitación con sus coterráneos ${ }^{18}$, los ucranianos con sus compatriotas y lo mismo sucede con los españoles, de forma que siguen permaneciendo límites en la socialización o fronteras simbólicas ${ }^{19}$ que

\footnotetext{
${ }^{16}$ Este criterio fue configurándose de modo implícito. Muchos estudiantes latinoamericanos preferían armar reuniones informales, noches de cine o pasar largas horas charlando en sus habitaciones con sus amigos. En este punto se importante diferenciar la postura arquitectónica de casa y la fenomenológica de hogar; cuando Pallasmaa (2016) discute sobre intimidad y hogar expresa que el hogar es el mediador entre el reino de lo público y de lo privado por lo que su transparencia es variable.

${ }^{17}$ El nombre "La Torre de Babel" intenta enaltecer la idea de multiculturalidad.

${ }^{18}$ El departamento está compuesto por dos ambientes que comparten dos personas. Cada piso tiene una cocina general. La conformación de los cuartos está prevista bajo la elección voluntaria de sus miembros, siendo posible la rotación cada vez que los estudiantes lo requieran.

${ }^{19}$ Lamont y Molnár (2002) proponen la distinción entre fronteras simbólicas y sociales como un modo de intentar comprender el rol de los recursos simbólicos (distinciones conceptuales, estrategias
} 
refuerzan la imbricación entre lugar y comunidad homogénea. Consecuentemente, el contacto entre las distintas culturas se ve forzado en los espacios de uso común donde se verifica el distanciamiento entre unos y otros. Los entrevistados expresaron sentir "incomodidad" durante el tiempo de uso de cocinas generales, los turnos de lavandería e incluso en el momento de compartir el ascensor. En alguno de estos sitios, el estruendo de las puertas cuando emergía el otro cultural, en forma de música u olor a comida desconocida, eran actos que aparecían. Por otro lado, el vínculo con los recepcionistas de la residencia estudiantil fue similar: el no saludo, la prohibición de ingreso a amigos que no vivían en la residencia, la falta de cooperación ante la gestión de un pago y la mirada indiferente al desandar los mismos pasillos fueron ejemplos brindados.

Estas fronteras invisibles parecen desaparecer o al menos marcan nuevos límites de contacto en lo que propone la noche o ante los encuentros fomentados por la universidad, como mencionamos. La diversión de la noche, en conjugación con el alcohol y la seducción, lleva a estos jóvenes a poder relacionarse con sus pares, desde otro ángulo. El poder de la globalización musical resulta eficiente y hace que los estudiantes estén más receptivos al intercambio. De hecho muchos latinoamericanos han podido entablar sus primeras conversaciones con estudiantes de otros orígenes en estos recintos.

\section{Repensando el lugar}

Para nuestros sujetos de análisis los procesos de socialización más intensos se forjaron entre los estudiantes de la misma región, es decir, entre los mismos latinoamericanos. En este sentido, cobra importancia la relación entre comunidad y lugar (Barros, 2000), particularmente porque la configuración del lugar en tanto porción afectiva se desprende de la sensación de comodidad que tiene impactos positivos en la subjetividad de los involucrados. Las disparidades que pueden suscitarse en las costumbres, la gastronomía, los regionalismos de la lengua, propias de la diversidad cultural de América Latina, han resultado secundarias frente a la fuerza de comunidad que los enlazó, posiblemente porque también compartían una misma situación de soledad y de vulnerabilidad en un país lejano no sólo geográfica sino culturalmente. Por ende, las trayectorias cotidianas de éstos seguían un patrón grupal, intentando simular rutinas de su lugar de origen o más específicamente de su hogar, como reunirse en una cafetería, ir al cine, festejar cumpleaños, cocinar comida típica y escuchar música latina. Siguiendo esta idea, desde el urbanismo Pallasmaa

interpretativas, tradiciones culturales) en la creación, mantenimiento, cuestionamiento e incluso disolución de diferencias sociales institucionalizadas (clase, género, etnia). Las fronteras simbólicas son distinciones conceptuales realizadas por los actores para categorizar objetos, gente, prácticas e incluso tiempo y espacio; muchas veces las fronteras simbólicas persisten cuando se han abolido las fronteras sociales. 
(2016) subraya la memoria del hogar como un escenario de rituales, ritmos personales, rutinas diarias en la gradual adaptación al mundo. Es así que podemos dejar evidenciado la importancia de la premisa de Relph (2009) en tanto el lugar puede estar centrado espacialmente en la escala local pero de ninguna manera se encuentra constreñido en ella, es decir, un lugar puede estar en cualquier escala. En este sentido, se producen las microescalas del lugar que portan los latinoamericanos y, en ocasiones, éstas friccionan con la escala local de pertenencia que encarnan los residentes polacos. Se trata de dimensiones mentales que pueden ir desde una cultura específica hasta la identidad nacional (Pallasmaa, 2016).

Por otro lado, Relph agrega que viajar ${ }^{20}$ sería enormemente difícil para la puesta en marcha de una consciencia del lugar porque nada sería familiar, nada nos pertenecería. En la búsqueda del espacio vivido, percibido, representado, experimentado (Lindón, 2007), Relph propone pensar que el sentido de lugar y el no lugar en términos de Augé, están unidos en una especie de abrazo geográfico que transita aspectos de ambos. Por ejemplo, los aeropuertos conforman un no lugar pero desde las experiencias citadas producen un efecto de sentido de lugar negativo. Por lo tanto, ¿los efectos de la experiencia vivida, siempre específica e individual, deberían ser el criterio fundante del no lugar?

Cuando Maltas (2015) evoca la noción de "topografía filosófica", a partir de la cual el lugar es una pieza clave para la comprensión del ser humano, es susceptible pensar que la extrañeza de la ciudad es lo que llevó, tempranamente, a estos estudiantes a congregarse mancomunadamente hasta conformar su lugar enraizado en una expresión que resonó en las múltiples entrevistas: "mi familia latina" ${ }^{21}$. En adición, Relph (2009) sugerirá una distinción entre "sentido de lugar" y "espíritu del lugar" que es útil para comprender mejor el caso. El sentido de lugar es la facultad mediante la cual captamos el espíritu del lugar y eso nos permite apreciar las diferencias y similitudes entre los lugares; espíritu de lugar existe principalmente fuera de nosotros, se experimenta a través de la identificación y la intención. El espíritu de lugar es lo que Pallasmaa asocia con el hogar, "un estado difuso y complejo que integra recuerdos e imágenes, deseos, miedos, pasado y presente" (2016:18). En cambio, el sentido de lugar está dentro de nosotros y se potencia ante similitudes que encontramos en lugares nuevos. Esta diferencia vivida significa que, si bien es posible diseñar entornos para mejorar el espíritu del lugar recreando patrones de vida, de ninguna manera se logra tan fácilmente construir un sentido de lugar. En efecto, Maltas (2015) fundamenta que en el sentido de lugar emerge la participación activa con ese lugar y el arraigo en las relaciones que lo hacen.

\footnotetext{
${ }^{20} \mathrm{El}$ acto de viajar será entendido desde una premisa profunda, interesada en construir vínculos con la sociedad local y no desde la perspectiva turística.

${ }^{21}$ Esta idea fue tan significativa que luego de la experiencia erasmus los estudiantes crearon un grupo de whatsapp con este nombre y siguen en contacto hasta la actualidad.
} 
Figura 4. Estudiantes latinoamericanos

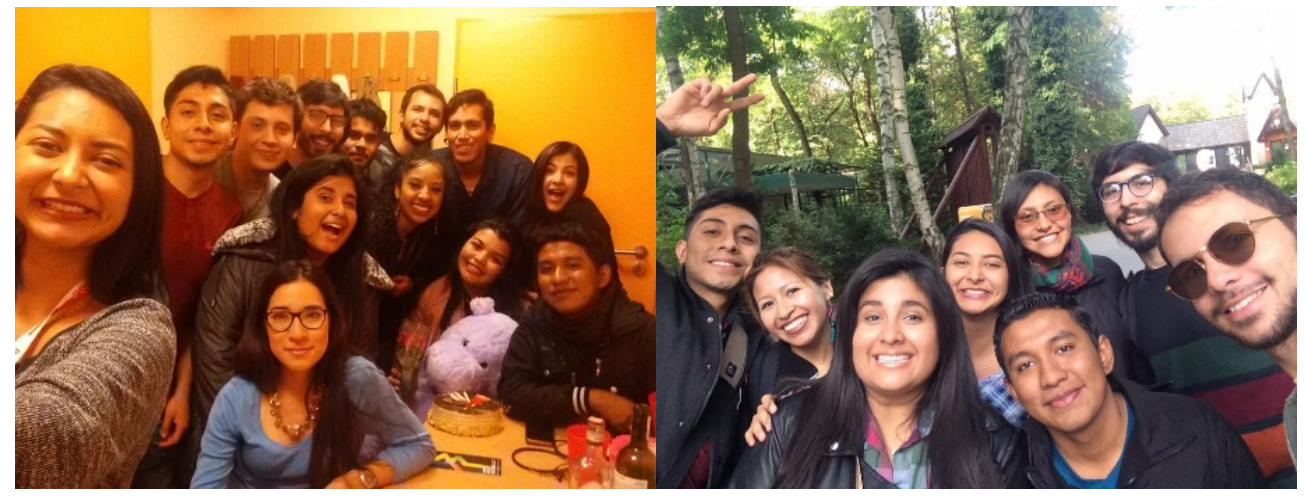

Fuente: Larreche, 2016.

Lo simbólico de mi familia latina da cuenta de la historia y del deseo de cada estudiante de reproducir un sentido de lugar que resulta dificultoso, principalmente por la temporalidad de la estancia. La dimensión temporal es vertebral en la construcción de los lugares (Tuan, 1996; Maltas 2015). Asimismo, el sentido de lugar alude a una localización auténtica de la vivencia ${ }^{22}$, aunque en este caso empírico la misma es perturbada por la tensión con el sentido de lugar-pertenencia que imponen los locales. Si bien estos estudiantes entraman las bases de un espíritu del lugar a través de las prácticas mencionadas nunca logran consolidar un sentido de lugar ya que la interiorización afectiva y la espontaneidad con el entorno cotidiano nunca se produce porque los avatares que se van presentando no les permiten despegarse de ese sentimiento de nostalgia que alude a su hogar natal.

\section{A modo de conclusión}

Los estudiantes latinoamericanos se introducen recientemente en los programas Erasmus, como parte de una espacialidad del conocimiento, en Lodz. Este hecho nos permite repensar la concepción del lugar desde la impronta geográfica. Se observa que el contexto geopolítico imperante de la Unión Europea condiciona los intercambios sociales, agudizando los parámetros negativos con los que se interpela a los extranjeros. Las tres espacialidades discutidas que incluyen las trayectorias por la

\footnotetext{
${ }^{22}$ Una auténtica actitud hacia el lugar comprende una directa y genuina experiencia de la identidad del lugar, no mediada ni distorsionada a través de una serie de arbitrarias modas sociales e intelectuales acerca de cómo debe ser experimentado, ni a través de convenciones estereotipadas (Relph, 1976: 64).
} 
ciudad, el espacio universitario y la residencia estudiantil permiten retratar una acepción del lugar particular y cambiante para los latinoamericanos.

El juego de inclusión y exclusión es un continuum en las vivencias de estos estudiantes. La adaptación al espacio se proyecta en la similitud cultural, de los gustos y preferencias, de la calidad de principiantes en el exterior pero también porque comparten la exposición a procesos de estigmatización en su espacio vivido. Por otro lado, estas dificultades se extienden al campus universitario y a los dorms pero son compensadas por la emergencia del espíritu de lugar expresado en la idea-fuerza $m i$ familia latina. En esta dirección la conformación del lugar se ancla en una microcomunidad a través de la reproducción de prácticas reconocidas por ese "nosotros".

Se ha demostrado que la movilidad vertical muchas veces resulta idealizada y que la movilidad horizontal se torna compleja, excepto cuando los estudiantes se asemejan en términos socioculturales. Esto puede ser efecto del contexto europeo actual y de la idiosincrasia polaca en particular que afecta el sentido de lugar de los estudiantes que se asumen como extranjeros.

La globalización educativa como fenómeno coyuntural conduce a plantear interesantes análisis que, confrontados con esta perspectiva de la geografía humana, nos acercan al espacio sentido de los sujetos en cuestión. Con este caso se pueden advertir las "ambivalencias de lo global" (Bhabha, 2013:19) en la negociación constante del reconocimiento de la alteridad: por un lado una integración puesta en marcha por convenios académicos y, por otro lado, la desintegración social en la experiencia concreta. Como consecuencia, el sentido de lugar de los recién llegados no reúne las condiciones para inspirar una actitud topofílica con el espacio de destino y se torna sensiblemente precario ante la xenofobia que se materializa en ciertas escenas del territorio.

Por lo expuesto, domina entonces una constante inestabilidad en la conformación del lugar en el marco de comunidades paradójicas (Bhabha, 2013) ya que estos estudiantes son actores fundamentales de las espacialidades del conocimiento pero su diversidad cultural es expuesta permanentemente. Finalmente, interiorizarse por aquellos estudiantes que no proceden de Europa desde un enfoque humanístico puede ser una herramienta de gran relevancia en la comprensión de los retos que supone el reconocimiento de la pluralidad. Como sostiene Pallasmaa: "el gran error del homo faber reside en su convencimiento de que el hombre puede existir sin un domicilio fijo, que la tecnología es capaz de transformar el mundo de modo que ya no sea necesario experimentarlo a través de las emociones" (2016:13).

\section{Bibliografía}

Barros, C. (2000): Reflexiones sobre la relación entre lugar y comunidad. Documents d'analisi geografica, 37, 81-94.

Bhabha, H. (2013): Nuevas minorías, nuevos derechos: notas sobre cosmopolitismos vernáculos. Buenos Aires, Siglo Veintiuno. 
Bótas, P. y Huisman, J. (2013): A Bourdieusian analysis of the participation of Polish students in the ERASMUS programme: cultural and social capital perspectives. Higher Education, 66, 741-754.

Bourdieu, P. (1991): El sentido práctico. Buenos Aires, Siglo Veintiuno.

Cairns, D. (2014): "I Wouldn't Stay Here": Economic Crisis and Youth Mobility in Ireland. International Migration, 52, 236-249.

Collins, F. L. (2009): Connecting "home" with "here": Personal homepages in everyday transnational lives. Journal of Ethnic and Migration Studies, 35, 839-859.

Deakin, H. (2014): The drivers to Erasmus work placement mobility for UK students. Children's Geographies, 12, 25-39.

González, C. R., Mesanza, R. B. y Mariel, P. (2011): The determinants of international student mobility flows: An empirical study on the Erasmus programme. Higher Education, 62, 413-430.

Guber, R. (2004): La entrevista antropológica: introducción a la no directividad y la entrevista antropológica: preguntas para abrir los sentido. En El salvaje metropolitano. Reconstrucción del conocimiento social en trabajo de campo. Buenos Aires, Paidós.

Iriondo, I. (2019): Evaluation of the impact of Erasmus study mobility on salaries and employment of recent graduates in Spain. Studies in Higher Education, 1-19.

King, R. y Raghuram, P. (2013): International student migration: mapping the field and new research agendas. Population, Space and Place, 19, 127-137.

Lamont, M. y Molnar, V. (2002): The study of boundaries in the social sciences. Annual review of Sociology, 28, 167-195.

Lindón, A. (2007): La ciudad y la ida urbana a través de los imaginarios urbanos. Eure, 33, 716.

Llurda, E., Gallego-Balsà, L., Barahona, C., y Martin-Rubió, X. (2016): Erasmus student mobility and the construction of European citizenship. The Language Learning Journal, 44, 323-346.

Malpas, J. (2012): Putting space in place: philosophical topography and relational geography. Environment and Planning D: Society and Space, 30, 226-242.

Malpas, J. (2015): Pensar topográficamente: lugar, espacio y geografía. Documents d'analisi geográfica, 61, 199-229.

Mendicoa, G. (2003): Sobre tesis y tesistas. Lecciones de Enseñanza-Aprendizaje. Buenos Aires, Espacio.

Monk, J. (2000): Looking out, looking in: The 'other' in the journal of geography in higher education. Journal of Geography in Higher Education, 24, 163-177.

Otero, M. S. (2008): The socio-economic background of Erasmus students: A trend towards wider inclusion? International Review of Education, 54, 135-154.

Pallasmaa, J. (2016): Habitar. Barcelona, Gustavo Gili.

Relph, E. (2009): A pragmatic sense of place. Environmental and architectural Phenomenology, 20, 24-31.

Santarelli, S., Campos, M. (2002): Corrientes epistemológicas, metodología y prácticas en geografía. Propuestas de estudio en el espacio local. Bahía Blanca, EDIUNS. 
Sautu, R., Boniolo, P., Dalle, P. y Elbert, R. (2005): Manual de metodología. Construcción del marco teórico, formulación de los objetivos y elección de la metodología. Buenos Aires, CLACSO.

Teichler, U. (2004): Temporary study abroad: the life of ERASMUS students. European Journal of Education, 39, 395-408.

Tuan, Y. (1977): Space and place: the perspective of experience. University of Minessota Press.

Tuan, Y. (1996): Space and Place: Humanistic Perspective. En Agnew, J.; Livingstone, D. y Rogers, A. (Eds.): Human Geography. An Essential Anthology. Oxford, Blackwell.

Vasilachis de Gialdino, I. (2006): Estrategias de investigación cualitativa. Barcelona, Gedisa. 